\title{
Sudden sensorineural hearing loss in children: Our experiences in tertiary care teaching hospital of eastern India
}

\author{
Santosh Kumar Swain'1, Mahesh Chandra Sahu², Jasashree Choudhury³ \\ 'Department of Otorhinolaryngology, IMS and SUM Hospital, Siksha "O" Anusandhan University, K8, Kalinganagar, \\ Bhubaneswar, Odisha, India \\ 2Directorate of Medical Research, IMS and SUM Hospital, Siksha "O" Anusandhan University, K8, Kalinganagar, Bhubaneswar, \\ Odisha, India \\ ${ }^{3}$ Department of Pediatrics, IMS and SUM Hospital, Siksha "O" Anusandhan University, K8, Kalinganagar, Bhubaneswar, Odisha, \\ India
}

\section{ABSTRACT}

Aim of the study: Sudden sensorineural hearing loss (SSNHL) is an uncommon clinical entity in children. The etiopathogenesis, clinical profile and management are not well understood and the incidence is not well known in medical literature. The goal of this study is to evaluate prevalence, etiology, treatment and outcome of SSNHL in the pediatric patients.

Material and methods: We had undertaken a retrospective study of the children below 18 years of age with SSNHL treated between July 2014 to August 2017. The data assessed were age, sex, clinical manifestations, onset of hearing loss, audiological profile, other diagnostic tools, treatment and outcome.

Results: The average age of the children presenting with SSNHL is 10.43 years (1-18 years). Twelve patients (6 right and 6 left) had unilateral hearing loss and 5 patients had bilateral hearing loss. Hearing loss ranges from profound (52.94\%) to mild and unknown etiology in $70.58 \%$ and viral $29.41 \%$. Hearing was completely improved in 5 cases (29.41\%), partially improved in 8 cases (47.05\%) and no improvement in 3 cases (17.64\%). When comparing oral with oral plus intratympanic (IT) steroid, hearing output is stastically significant ( $p=0.04020$ ) with 95\% confidential level is 0.69 to 0.02 and $t=2.1381$.

Conclusions: The exact incidences of SSNHL in pediatric age groups are not established in medical literature. Pediatric patients are usually delayed presentations and associated with unknown etiology in majority of cases. SSNHL has serious impact on life and it should be follow up for preventing morbidities and for starting rehabilitation program as early as possible.

\section{KEY WORDS:}

sudden sensorineural hearing loss, children, tinnitus, steroid.

\section{INTRODUCTION}

Sudden sensorineural hearing loss (SSNHL) is a rare clinical entity in children. Sudden hearing loss was first documented by de Kleyn in 1944 and defined as hearing loss of at least 30 decibel $(\mathrm{dB})$ in three consecutive frequencies in the standard pure tone audiogram over three days or less [1]. SSNHL in children is considered as an

\section{ADDRESS FOR CORRRESPONDENCE:}

Santosh Kumar Swain, Department of Otorhinolaryngology, IMS and SUM Hospital, Bhubaneswar, Odisha, India, e-mail: santoshvoltaire@yahoo.co.in 
otologic emergency and rarely reported in medical literature. The incidence of SSNHL ranges between 10.7 and 27 per 100,000 persons per year [2]. The incidence of SSNHL increases with age, with 8 per 100,000 under the age of 18 years and 70 per 100,000 over 65 years [2]. Early treatment is necessary for preventing permanent hearing loss. This is important in children as it affect speech and hearing if not improved which also affect academic and social performance. Often the children are unable to report their hearing loss, which may be not detected in acute stage when treatment is the most effective. The degree of hearing loss has an effect on the rate of recovery [3]. The causes of SSNHL in adults are usually idiopathic whereas the idiopathic cases are rare in children due to the small size of the available series. Presently the treatment protocol of SSNHL is available which include systemic steroids as primary treatment and intratympanic steroid administration when there is no improvement after systemic steroid treatment or contraindications for systemic steroid [4]. This study discuss about prevalence, etiology, clinical profile and outcome of SSNHL among children in a tertiary care teaching hospital of eastern India.

\section{MATERIAL AND METHODS}

We performed a retrospective study of patients below 18 years of age between July 2014 to August 2017 with sudden sensorineural hearing loss. This study was approved by institutional ethical committee (IEC). The gap between sudden onsets of hearing loss and starting the treatment was within two weeks. All the children depending on the patient's age and developmental level, the testing methodology like visual reinforcement audiometry, conditioned play audiometry and conventional pure tone audiometry. The normal hearing is defined at $\leq 20 \mathrm{~dB}$. In our study, the degree of hearing loss ranges from mild sensorineural hearing loss to profound loss across frequencies. The degree of hearing loss was determined as mild (20-40 dB), moderate (40-60 dB), moderate to severe (60-75 dB), severe (75-90 dB) and profound (>90 dB). All patients with sudden hearing loss had done pure tone audiometry at frequencies between $250 \mathrm{~Hz}$ to $8000 \mathrm{~Hz}$ and had to be at least more than $20 \mathrm{~dB}$ in 3 consecutive frequencies in comparison to the unaffected ear. All children reported abrupt onset of hearing loss. All the children with hearing loss were treated with steroid. Eleven cases were treated with oral prednisolone given at an initial dose of $3 \mathrm{mg} / \mathrm{kg}$ body weight. The dose of the prednisolone was reduced every second day and for the maximum of 14 days or completed 2 days after the hearing had normalized in pure tone audiometry. In few cases, intravenous (IV) hydrocortisone $1 \mathrm{mg} / \mathrm{kg}$ /day given in divided doses. Whenever there was no improvement of hearing after systemic therapy or contraindication for steroid therapy, intratympanic injection of steroid like dexamethasone $1 \mathrm{mg}$ every 12 hour for 7 days was given through grommet inserted into the tympanic membrane. After intratympanic injection of steroid, children were positioned at the non-affected side for 3 minutes. All intratympanic injections were done under all aseptic precautions. Another pure tone audiometry was performed 2 to 3 weeks after therapy and combined with brainstem evoked response audiometry. The follow up for all children were done at 3 months, 6 months and 1 year. Complete hearing improvement was defined as hearing level is similar to the non-affected ear, partial hearing improvement is defined when more than $10 \mathrm{~dB}$ in at least one frequency and no improvement of hearing when there is no change in hearing in audiogram after treatment. In our study, data recorded are age, gender, symptoms associated with hearing loss like aural fullness, tinnitus, vertigo, and otalgia. Onset of hearing loss, audiological results, serological results, imaging, treatment and its outcome were documented.

\section{RESULTS}

We diagnosed 17 children with SSNHL where 11 were male and 6 were female child. All children were healthy and without any family history of hearing loss. All were presented with abrupt onset of hearing loss. Mean age was 9.64 years (1-18 years). The right ear was affected in $6(35.29 \%)$ children, the left ear in $6(35.29 \%)$ and both ears in $5(29.41 \%)$ children. Fourteen children $(82.35 \%)$ complained other symptoms in addition to hearing loss and most common one was tinnitus. Three patients complained otalgia (17.64\%), two patients $(11.76 \%)$ complained vertigo and one $(5.88 \%)$ presented herpetic lesions but serology for herpes simplex was negative. There was higher incidence of profound hearing loss in 9 cases $(52.94 \%)$, severe hearing loss in 3 cases (17.64\%), moderate hearing loss in 3 cases (17.64\%) and mild in 2 cases $(11.76 \%)$. Initial treatment was oral steroids in 11 cases $(64.70 \%)$, IV steroids in 6 cases $(35.29 \%)$ and 6 cases $(35.29 \%)$ undergone intratympanic steroids along with 6 oral steroids. The systemic steroids was continued for $1-2$ weeks (median $=7$ days). In three cases, parents of children decided to discontinue this treatment due to fear of side effects. Intratympanic steroids are given in 6 cases $(35.29 \%)$ and lasted between 6 and 8 days (mean $=7$ ). One patient stopped IT treatment after 4 days. There were no adverse effects following either systemic or IT steroid treatment. Non-contrast CT scan of temporal bone in 5 patients and MRI in 11 patients were within normal limits. None of the imaging revealed any pathology. Serological study revealed cytomegalovirus (CMV) in 3 cases, Epstein-Barr virus (EBV) IgM in one, EBV IgM in 1 case. Hearing was completely improved in 5 cases (29.41\%), partially improved in 8 cases $(47.05 \%)$ and there was no improvement in 3 cases (17.64\%) (Table 1). There is no statistically significant between oral and IV steroid ( $p=0.729$ ) but when comparing oral with oral 
TABLE 1. Clinical and audiological profile of the children

\begin{tabular}{|c|c|c|c|c|c|c|c|}
\hline Serial No & Age (years) & Gender & Side of the ear & $\begin{array}{c}\text { Day of the treatment } \\
\text { started }\end{array}$ & Other symptoms & Steroids & Outcome \\
\hline 1 & 7 & Male & Right & 2 & Tinnitus & Oral & $\mathrm{PI}$ \\
\hline 2 & 3 & Male & Right & 4 & Tinnitus, fullness & Oral & $\mathrm{PI}$ \\
\hline 3 & 11 & Female & Bilateral & 0 & Tinnitus & IV & $\mathrm{Cl}$ \\
\hline 4 & 5 & Male & Left & 3 & Otalgia & Oral, IT & $\mathrm{PI}$ \\
\hline 5 & 18 & Male & Right & 7 & Tinnitus & Oral, IT & $\mathrm{PI}$ \\
\hline 6 & 9 & Female & Left & 1 & Tinnitus & IV & $\mathrm{PI}$ \\
\hline 7 & 1 & Female & Left & 20 & Tinnitus & Oral & $\mathrm{NI}$ \\
\hline 8 & 8 & Male & Bilateral & 5 & Vertigo & Oral & $\mathrm{NI}$ \\
\hline 9 & 12 & Male & Right & 3 & Tinnitus & IV & $\mathrm{Cl}$ \\
\hline 10 & 14 & Male & Bilateral & 2 & Tinnitus & Oral, IT & $\mathrm{Cl}$ \\
\hline 11 & 4 & Male & Left & 32 & Tinnitus, fullness & Oral & $\mathrm{NI}$ \\
\hline 12 & 7 & Female & Right & 4 & Tinnitus & Oral & $\mathrm{PI}$ \\
\hline 13 & 9 & Female & Bilateral & 1 & Vertigo & IV & $\mathrm{PI}$ \\
\hline 14 & 12 & Male & Left & 0 & Tinnitus, otalgia & IV,IT & $\mathrm{PI}$ \\
\hline 15 & 13 & Female & Left & 1 & Tinnitus & IV & $\mathrm{PI}$ \\
\hline 16 & 16 & Male & Bilateral & 2 & Herpetic lesions, otalgia & Oral, IT & $\mathrm{Cl}$ \\
\hline 17 & 15 & Male & Right & 0 & Tinnitus & Oral, IT & $\mathrm{Cl}$ \\
\hline
\end{tabular}

IV - intravenous; IT - intratympanic; $\mathrm{Pl}$ - partial improvement; $\mathrm{Cl}$ - complete improvement; $\mathrm{NI}$ - no improvement

plus IT steroid, it is statically significant $(p=0.04020)$ with $95 \%$ confidential level is 0.02 to 0.69 and $t=2.1381$. The statical analysis evaluated by SPSS 20 software. One patient was lost follow up.

\section{DISCUSSION}

Sudden sensorineural hearing loss (SSNHL) is an otological emergency in medicine. SSNHL in children is a rare clinical entity and less frequently discussed in the medical literature and the exact incidence is not documented. The causes of SSNHL in adults may be due to meningitis, labyrinthitis, temporal bone trauma, autoimmune disorders, retro-cochlear pathology, ototoxic medications, central nervous lesions and stroke [5]. However in majority of cases the cause is not clearly identified and is presumed to be vascular insults or infections [6]. In pediatric patients, the SSNHL is less likely by vascular insults and retro-cochlear pathology [7]. Majority of the cause for SSNHL is idiopathic in this study. One study reported Lyme disease and EBV are leading cause for SSNHL [7]. Congenital cytomegalovirus, radiographic findings of enlarged vestibular aqueduct and non-organic hearing loss are other causes and not common etiology in adult patients. In present study, no etiology was found in $70.58 \%$ of the subjects. Adult patients with SSNHL usually complain hearing loss much earlier than children and sometimes present with associated symptoms like tinnitus, vertigo and otalgia. Our patients in this study also presented with similar associated symptomatology. However, these presentations may be delayed in pediatric age groups. The delay in presentation will delay the treatment and can affect the outcome of the treatment. Hearing loss at very early age of life affects speech and language. It also affects academic and social performance in life. The diagnosis of SSHNL sometimes difficult particularly at a very young age, therefore a high index of suspicion is needed. The role imaging in SSNHL is not well defined and it does not have impact on the treatment. However to level the SSNHL to idiopathic, MRI is needed to rule out acoustic neuroma as it is seen in 2\% cases of SSNHL and it is associated with partial recovery [8]. Currently American Academy of Otolaryngology guidelines recommended MRI for SSNHL because sudden hearing loss are associated with a retro-cochlear lesions in MRI [9]. The MRI is important diagnostic tool to rule out acoustic neuroma, which is not common in pediatric patients [9]. There is still a debate in pediatric literature whether to use CT scan or MRI for diagnosis of sensorineural hearing loss [10]. Serial audiometric testing are done along with the imaging for documenting progression of hearing loss. All patients had undergone pure tone audiometry at the time of first visit and next after completion of the treatment. Auditory brainstem response (ABR) in children is done to rule out non-organic hearing loss [11]. Laboratory testing were not done in our patients. Routine laboratory evaluation has low diagnostic yield and should only performed in case clinical suspicion [12]. It has seen that severe 
hearing loss has poor prognostic factor [13]. Treatment of SSNHL is a subject of debate. Presently the available treatment options are systemic steroids, intratympanic steroids, both systemic and intratympanic steroids and hyperbaric oxygen therapy. The drugs like anti-viral, vasodilators, thrombolytic, vasoactive substance and antioxidants are not usually advocated in the treatment of SSNHL [6]. Oral steroids are usually given in the initial treatment for SSNHL. In one study, showed efficacy of oral steroids with $61 \%$ of patients improved with oral steroids whereas only $32 \%$ improved with placebo [14]. Intratympanic injections of steroid is another alternative to the oral steroid. Intratympanic steroid provides localized treatment with higher concentration at perilymph and avoid systemic side effects. Few complications may occur like otalgia during and after treatment, tympanic membrane perforation, dysgeusia, vertigo and transmission of infection to the middle ear. It has been seen that oral and intratympanic steroid treatments have equally effacious in the treatment of SSNHL [15]. Gundogan et al., showed better hearing outcome with both oral and intratympanic steroids than either one alone [16]. Hyperbaric oxygen therapy (HBOT) is another treatment option in medical literature. HBOT decreases the edema and hypoxia and may help with immune response to ischemia and infection [17]. It may be helpful in case of failed traditional treatment options [18]. The combined treatment of steroids and $\mathrm{HBOT}$ has better outcomes in comparison to medical treatment alone [19]. HBOT is costly and has potential adverse effects and power of the many studies is low. None of our patients under went HBOT in present study. The rate of recovery of hearing in case SSNHL among children varies between the different studies. In one study with 14 patients under the age of 18 years treated with IV prednisolone, the rate of complete recovery was $57 \%$ whereas the partial recovery rate was $36 \%$ [20]. Tarshish et al. showed complete recovery in $10 \%$ cases and some improvement in 10\% cases after treatment with systemic steroids and without intratympanic steroids [21]. In our study, hearing completely improved in 5 cases (29.41\%) and partial improved in 8 cases $(47.05 \%)$ and no improvement in 3 cases (17.64\%). Therefore, more such studies are required to elaborate on the recovery rate from SSNHL in children. When comparing recovery rate with adults, the complete recovery is higher and no improvement rate is significantly lower in children than adults [3]. In both pediatric and adult populations, hearing recovery is significantly higher in patients with mild SSNHL compared to profound SSNHL, but in children with moderate hearing loss, the rate of recovery is higher in comparison to adults, revealing that age is associated with a poorer prognosis [22]. The lower incidence of SSNHL in children is seen medical literature and it is still not clear work-up and treatment giving successful outcome for this population. The etiological role of viral infections is still not clear and it seems that pediatric CMV infection may play role in SSHNL. Similar to adult patients, intratympanic steroids may be considered for the treatment along with systemic corticosteroids.

\section{CONCLUSIONS}

SSNHL in children is a rare clinical entity. This study showed that the most common etiology of SSNHL in pediatric age group is idiopathic. Detail history taking and serological evaluations for ruling out viral etiology are important for diagnosis and treatment. Most of the children in our study showed improvement in hearing. Intratympanic steroid has additional benefit to the patient along with systemic steroids. A larger study is needed to establish the standardize the diagnosis and treatment. The diagnosis of SSNHL should be prompt and time waste can be prevented by not doing unnecessary investigations. SSNHL has serious impact on life and it should be follow up for preventing morbidities and for starting rehabilitation program as early as possible.

This study is limited by its retrospective nature and the small number of patients. However, it provides further data on this subject.

\section{DISCLOSURE}

The authors declare no conflict of interest.

\section{REFERENCES}

1. Benjamin ES, Charlotte A, Dorian OH, et al. Sudden sensorineural hearing loss. Lancet 2010; 375: 1203-1211.

2. Alexander H, Harris JP. Incidence of sudden sensorineural hearing loss. Otol Neurotol 2013; 34: 1586-1589.

3. Na SY, Kim MG, Hong SM, et al. Comparison of sudden deafness in adults and children. Clin Exp Otorhinolaryngol 2014; 7: 165-169.

4. Dedhia K, Chi DH. Pediatric sudden sensorineural hearing loss: Etiology, diagnosis and treatment in 20 children. Int J Pediatr Otorhinolaryngol 2016; 88: 208-212.

5. Khairi MD, Noor RM, Rahman NA, et al. The effect of mild hearing loss on academic performance in primary school children. Int $\mathrm{J}$ Pediatr Otorhinolaryngol 2010; 74: 67-70.

6. Kakehata S, Sasaki A, Futai K, et al. Daily short-term intratympanic dexamethasone treatment alone as an initial or salvage treatment for idiopathic sudden sensorineural hearing loss. Audiol Neurootol 2011; 16: 191-197.

7. Jecmenica J, Bajec-Opancina A. Sudden hearing loss in children. Clin Pediatr (Phila) 2014; 53: 874-878.

8. Fetterman BL, Saunders JE, Luxford WM. Prognosis and treatment of sudden sensorineural hearing loss. Am J Otolo 1996; 17: 529-536.

9. Suzuki H, Tabata T, Koizumi H, et al. Prediction of hearing outcomes by multiple regression analysis in patients with idiopathic sudden sensorineural hearing loss. Ann Otol Rhinol Laryngol 2014; 123: 821-825.

10. McClay E, Booth TN, Parry DA, et al. Evaluation of pediatric sensorineural hearing loss with magnetic resonance imaging. Arch Otolaryngol Head Neck Surg 2008; 134: 945-952.

11. Morita S, Suzuki M, Iizuka K. Non-organic hearing loss in childhood. Int J Pediatr Otorhinolaryngol 2010; 74: 441-446. 
12. Preciado DA, Lim LH, Cohen AP, et al. A diagnostic paradigm for childhood idiopathic sensorineural hearing loss. Otolaryngol Head Neck Surg 2004; 131: 804-809.

13. Bullo F, Tzamtzis S, Tirelli G. Update on the sudden hearing loss. Indian J Otol 2013; 19: 95-99.

14. Chen CY, Halpin C, Rauch SD. Oral steroid treatment of sudden sensorineural hearing loss: a ten year retrospective analysis. Otol Neurotol 2003; 24: 728-733.

15. Rauch SD. Idiopathic sudden sensorineural hearing loss. N Engl J Med 2008; 359: 833-840.

16. Gundogan O, Pinar E, Imre A, et al. Therapeutic efficacy of the combination of intratympanic methylprednisolone and oral steroid for idiopathic sudden deafness. Otolaryngol Head Neck Surg 2013; 149: 753-758.

17. Stachler RJ, Chandrasekhar SS, Archer SM, et al. Clinical practice guideline: sudden hearing loss. Otolaryngol Head Neck Surg 2012; 146: 1-35.

18. Horn CE, Himel HN, Selesnick SH. Hyperbaric oxygen therapy for sudden sensorineural hearing loss: a prospective trial of patients failing steroid and antiviral treatment. Otol Neurotol 2005; 26: 882-889.

19. Narozny W, Sicko Z, Przewozny T, et al. Usefulness of high doses of glucocorticoids and hyperbaric oxygen therapy in sudden sensorineural hearing loss treatment. Otol Neurotol 2004; 25: 916-923.

20. Chen YS, Emmerling O, Ilgner J, et al. Idiopathic sudden sensorineural hearing loss in children. Pediatr Infect Dis J 2005; 69: 817 821.

21. Tarshish Y, Leschinski A, Kenna M. Pediatric sudden sensorineural hearing loss: diagnosed causes and response to intervention. Int J Pediatr Otorhinolaryngol 2013; 77: 553-559.

22. Pitaro J, Bechor-Fellner A, Gavriel H, et al. Sudden sensorineural hearing loss in children: Etiology, management, and outcome. Int J Pediatr Otorhinolaryngol 2016; 82: 34-37. 